\title{
VULNERABILIDADES SOCIOECONÔMICAS E O COTIDIANO DA ASSISTÊNCIA DE ENFERMAGEM PEDIÁTRICA: RELATO DE ENFERMEIRAS
}

\author{
Socio-Economic Vulnerability and Pediatric Nursing Care \\ Routine: Nurses Report \\ Vulnerabilidad Socioeconómica y el cuidado diario de la \\ Enfermería Pediátrica: Informe de Enfermeras
}

Maria de Lourdes Rodrigues Pedroso ${ }^{1}$

Maria da Graça Corso da Motta²

\begin{abstract}
RESUMO
A equipe de saúde, quando considera as vulnerabilidades em que a criança e sua família estão inseridas, pode planejar e executar um cuidado integral a estas. Este estudo objetivou compreender as percepções dos enfermeiros sobre as influências das vulnerabilidades socioeconômicas no cuidado à criança e sua família, no ambiente de Unidades de Internação Pediátricas de um Hospital Universitário no Município de Porto Alegre. Trata-se de uma pesquisa qualitativa na perspectiva de um estudo exploratório descritivo. A coleta de informações ocorreu com nove participantes por meio de entrevistas individuais semiestruturadas, analisadas conforme referencial da Análise de Conteúdo. Emergiram três categorias de análise, duas das quais serão aqui exploradas: Vulnerabilidades socioeconômicas e o cotidiano da assistência de enfermagem pediátrica; Propostas e alternativas para o manejo das situações de vulnerabilidades socioeconômicas. Os resultados forneceram exemplos práticos e apontaram soluções para as vulnerabilidades socioeconômicas relacionadas com a assistência de enfermagem à criança e sua família.
\end{abstract}

Palavras-Chave: Vulnerabilidade. Enfermagem Pediátrica. Família.

\begin{abstract}
By considering the vulnerabilities faced by a child and their family, a health care team can plan and execute an integrated plan to address these issues. The objective of this study, conducted in the environment of pediatric units at a University Hospital in the city of Porto Alegre, is aimed at understanding the perception of nurses regarding the influence of socioeconomic vulnerabilities on the care of a child and their family. This qualitative research is performed in the context of a descriptive exploratory study. Data collection was conducted using semistructured, individual interviews with nine participants, and then analyzed consistent with content analysis references. Three categories emerged from the analysis, and two are explored in this study: the impact of socio-economic vulnerabilities on the pediatric nursing care routine; and, proposals and alternatives for management of situations resulting from socioeconomic vulnerabilities. The results provide practical examples and solutions to socioeconomic vulnerabilities as related to the nursing care for children and their families.
\end{abstract}

Keywords: Vulnerability. Pediatric nursing. Family.

\section{Resumen}

El equipo de salud, cuando considera la vulnerabilidad en la cual están inmersos el niño y su familia, puede planificar y brindarles un cuidado integral. Este estudio tuvo como objetivo comprender la percepción que tienen los enfermeros sobre la influencia de la vulnerabilidad socioeconómica, en el cuidado del niño y la familia, en el ámbito de las Unidades de Internación Pediátrica en un Hospital Universitario en el Municipio de Porto Alegre. Se trata de una investigación cualitativa desde la perspectiva de un estudio explorable descriptivo. La recopilación de las informaciones se realizó con nueve participantes a través de entrevistas individuales semiestructuradas, analizadas según referencial del Análisis de Contenido. Surgieron tres categorías de análisis, de las cuales dos son analizadas aquí: Vulnerabilidad socioeconómica y cuidado cotidiano de la enfermería pediátrica; Propuestas y alternativas para el manejo de situaciones de vulnerabilidad socioeconómica. Los resultados proporcionaron ejemplos prácticos e ofrecieron soluciones para solventar la vulnerabilidad socioeconómica vinculada al cuidado que brinda la enfermería al niño y su familia.

Palabras Clabe: Vulnerabilidade. Enfermería pediátrica. Família.

\footnotetext{
${ }^{1}$ Mestranda do Programa de Pós-Graduação em Enfermagem da Escola de Enfermagem da Universidade Federal do Rio Grande do Sul. Enfermeira Especialista em Enfermagem Pediátrica (EENF-UFRGS). Brasil. E-mail: malupedroso@gmail.com, ${ }^{2}$ Doutora em Filosofia da Enfermagem pela Universidade Federal de Santa Catarina. Professora Assistente do Departamento de Enfermagem Materno-Infantil da Escola de Enfermagem da Universidade Federal do Rio Grande do Sul. Docente Permanente do Programa de Pós-Graduação em Enfermagem (EENF-UFRGS). Brasil. E-mail: mottinha@enf.ufrgs.br
} 


\section{INTRODUCÃO}

Muitas são as vezes em que o cuidado integral da equipe de saúde encontra barreiras que estão relacionadas às vulnerabilidades da criança e de sua família. Entende-se que a vulnerabilidade pode ser baseada em três eixos: social, programático ou institucional e individual. ${ }^{1}$

0 eixo social inclui condições sociais e econômicas, acesso à informação, à educação, à assistência social e à saúde, a garantia de respeito aos direitos humanos e a situação sociopolítica e cultural do indivíduo. 0 eixo programático ou institucional associa-se a programas voltados especificamente para a prevenção, controle e assistência de agravos à saúde. 0 eixo individual refere-se ao acesso a recursos que possibilitam a adoção de comportamentos seguros ou saudáveis. Este último eixo está intrinsecamente relacionado com os eixos social e programático.

Acredita-se que a equipe de saúde, quando considera as vulnerabilidades em que a criança e sua família estão inseridas, pode planejar e executar um cuidado integral à saúde, favorecendo, desta forma, a identificação e a construção de redes de apoio, tanto em nível hospitalar quanto domiciliar. Percebe-se que quando os pais ou familiares recebem este suporte dos profissionais da saúde, por vezes, alteram suas atitudes em relação ao cuidado à saúde da criança, propiciando que a equipe de saúde alcance resultados que podem ir muito além do que é esperado.

0 referencial de ambiente ecológico, conforme a Teoria Ecológica de Urie Brofenbrenner, oferece um suporte para a compreensão do contexto em que as crianças e suas famílias estão inseridas, bem como suas influências no processo de saúde-doença. 0 ambiente ecológico é concebido como uma série de estruturas encaixadas, uma dentro da outra. No nível mais interno está o ambiente imediato que contém a pessoa em desenvolvimento, como, por exemplo, o lar. No próximo nível encontra-se não só o ambiente simples, mas as relações entre eles; no terceiro nível, invoca-se a hipótese de que o desenvolvimento é afetado por ambientes nos quais a pessoa nem sequer está presente e, em último nível, traz-se a ideia de toda a grande organização cultural humana. ${ }^{2}$

Na situação atual em que vivemos, o ciclo de vida normal de uma família pode sofrer desvios, em razão de muitos fatores. 0 desemprego, a miséria, a falta de moradia, a educação inacessível, a desinformação, o afastamento da mãe por um longo tempo, entre outros, são fatores que podem levar a um "desvio da saúde familiar". 3
A partir destas reflexões, salienta-se a importância de estabelecer um estudo que se proponha a voltar o olhar para a percepção dos enfermeiros sobre as dificuldades socioeconômicas que as famílias destas crianças apresentam, bem como suas implicações na prática terapêutica, desta forma procurando oferecer subsídios para uma prática assistencial contextualizada.

Neste artigo propõe-se a apresentação de duas categorias que compõem os resultados da pesquisa intitulada "Influências das Vulnerabilidades Socioeconômicas no Cuidado as Crianças em Unidades de Internação Pediátricas: A Visão do Enfermeiro" ${ }^{\prime \prime}$, que teve por objetivo compreender as percepções dos enfermeiros sobre as influências das vulnerabilidades socioeconômicas no cuidado à criança e seus familiares no ambiente de Unidades de Internação Pediátricas.

\section{METODOLOGIA}

Trata-se de uma pesquisa qualitativa, desenvolvida na perspectiva de um estudo exploratório descritivo, realizada em quatro Unidades de Internação Pediátricas do Hospital de Clínicas do Município de Porto Alegre, Rio Grande do Sul, que prestam atendimento a crianças em situações de agravos à saúde, mais especificamente: Unidade de Internação Pediátrica Nor te $-10^{\circ} \mathrm{N}$, Unidade de Internação Pediátrica Sul $-10^{\circ} \mathrm{S}$, Hematologia e Oncologia Pediátrica (Unidade de Oncologia Pediátrica $3^{\circ} \mathrm{L}$ ) e Unidade de Terapia Intensiva Pediátrica - UTIP.

As participantes foram nove enfermeiras que exercem suas atividades nessas Unidades, sendo que, como critério de inclusão, foi considerado o tempo de atividade exercido nestas Unidades, que deveria ser de no mínimo um ano. 0 número de participantes foi considerado suficiente, usando-se o critério da saturação das informações. ${ }^{5}$

A coleta de informações ocorreu por meio de entrevista individual semiestruturada, composta de três questões norteadoras versando sobre a temática das vulnerabilidades socioeconômicas, realizadas em ambientes apropriados das Unidades de Internação que serviram como campo do estudo, com o auxílio de um equipamento de áudio para a gravação das mesmas. Estas foram analisadas conforme referencial de Análise de Conteúdo. ${ }^{6}$ Esta etapa estabelece uma compreensão dos dados coletados, confirma ou não os pressupostos da pesquisa e amplia o conhecimento sobre 0 assunto pesquisado, articulando-o ao contexto cultural do qual faz parte. ${ }^{6}$ 
Essa análise ocorreu a partir das seguintes fases: préanálise - fase em que ocorreu a organização e preparação do material, a partir de leitura flutuante; exploração do material - etapa de conclusão da preparação do material e denominação das categorias; tratamento e interpretação dos dados obtidos - descrição das categorias evidenciadas e posterior interpretação.

A coleta de informações teve início após aprovação do projeto de pesquisa pela Comissão de Pesquisa da Escola de Enfermagem - UFRGS e Comitê de Ética em Pesquisa do Grupo de Pesquisa e Pós-Graduação do Hospital de Clínicas de Porto Alegre, onde foi registrado sob o protocolo $n^{0} 08-411$. Às informantes selecionadas foi apresentado um Termo de Consentimento Livre e Esclarecido, informando os objetivos do estudo e que este não apresentava riscos, bem como sobre o caráter voluntário de suas participações com possibilidade de desistência a qualquer momento, sem qualquer prejuízo às suas pessoas e garantia de anonimato. Os dados utilizados serão guardados por cinco anos, conforme legislação específica regulamentadora de direitos autorais n. 9610 de 1998 para pesquisas desta natureza.

\section{RESULTADO E DISCUSSÃO}

A partir da análise dos dados qualitativos, emergiram três categorias, e duas delas serão exploradas neste relato: Vulnerabilidades socioeconômicas e o cotidiano da assistência de enfermagem pediátrica; e Propostas e alternativas para o manejo das situações de vulnerabilidades socioeconômicas.

\section{Vulnerabilidades socioeconômicas e o cotidiano da assistência de enfermagem pediátrica}

Vários são os símbolos e expressões corporais e comportamentais que indicam ao enfermeiro e à equipe multidisciplinar que determinada família está exposta a fatores que podem desencadear situações de vulnerabilidade. Alguns destes sinais são facilmente perceptíveis por aqueles que prestam o cuidado à criança e seus familiares, outros se encontram velados, por diversos elementos que dificultam a sua apresentação e interpretação.

Estes aspectos aparecem nas falas dos participantes sobre diversas temáticas, sendo as mais frequentes: 0 acesso aos serviços de saúde, as condições de moradia das crianças e suas famílias e a dificuldade de compreensão das orientações fornecidas pela equipe de saúde acerca do diagnóstico e tratamento das patologias.

Em um cenário de miserabilidade e condições de existência sem dignidade, estão inseridas as trajetórias de vida de muitas crianças e famílias que hoje enfrentam uma hospitalização nos diversos serviços de saúde espalhados pelo país. Mesmo diante de muitas dificuldades, os serviços de saúde constituem-se em local de segurança para as mães que trazem seus filhos nos braços, em muitos casos gravemente enfermos, após uma longa jornada, visando um adequado atendimento. Esta busca, que para muitos pais é árdua, cansativa e dolorosa, aparece como exemplo claro de como a situação econômica de uma família está, em nossa sociedade atual, intimamente ligada à oferta e rapidez de acesso aos recursos e serviços de saúde e à qualidade dos mesmos. É o que nos ilustra a fala a seguir:

[...] Crianças que chegam até nós, que, por exemplo, tiveram a dificuldade de ter um diagnóstico definido e foram em vários serviços de saúde e chegaram no hospital com uma situação de saúde muito vulnerável, por exemplo, crianças com problemas respiratórios graves e com derrame pleural que passaram por vários serviços e foram Ihe traçados tratamentos supérfluos, ou talvez, não tiveram a investigação que mereciam. Isto eu acho que é uma das coisas marcantes que eu percebo no meu cotidiano profissional [...] (E.2).

A dificuldade de acesso aos serviços de saúde se constitui como importante marcador de vulnerabilidade. 7 Vulneráveis são todos os que perderam seus direitos ou que os têm ameaçados nas condições de sua realização. Então, se crianças e adolescentes são, por um lado, fragilizados pela situação social, que representa uma ameaça ao seu destino (o problema da exclusão social), por outro existem também os conflitos provenientes do convívio social que ameaçam o seu bem-estar. Ou seja, elas não sofrem apenas da falta de acesso às instituições e serviços, mas também da qualidade de interação com os adultos. ${ }^{8}$ A seguinte fala de uma participante nos demonstra este quadro:

[...] 0 que eu penso que sejam vulnerabilidades sociais são a própria caminhada até chegar ao serviço [...]. Por não terem um acesso diferente de chegar até o serviço de saúde elas dependem muito do que é dito, do que Ihes é explicado para chegar a esse serviço. Muitas vezes, não têm dinheiro para o transporte, não têm condições socioeconômicas que Ihe permitam encontrar esse acesso mais fácil. Não tem conhecimento do funcionamento e não somente a falta de dinheiro em si, mas a falta de conhecimento da rede social. Falta uma rede social organizada para chegar a esse acesso [...] (E.6). 
Ter os olhos fixos para a realidade de que os caminhos ainda são obscuros e tortuosos para muitos é responsabilidade das políticas de saúde, que prezam por sua eficácia, mais especificamente dos que as constroem no dia-a-dia, seja no âmbito hospitalar, seja no que tange à Saúde Coletiva, como um todo.

Quando nos referimos a este âmbito das responsabilidades dos serviços, podemos evocar o conceito de Vulnerabilidade Programática. ${ }^{9} 0$ componente institucional ou programático da vulnerabilidade conecta os componentes individuais e sociais. Estes envolvem o grau e qualidade de compromisso, recursos, gerência e monitoramento de programas, nacionais, regionais ou locais de prevenção e cuidado, os quais são importantes para identificar necessidades, canalizar os recursos sociais existentes e otimizar seu uso. 0 componente programático nos orienta a situar as dimensões educativas para além do caráter normativo e centrado no objeto, levando-nos a articular as intervenções em saúde e nas ações programáticas e, principalmente, a repensar o cuidado em saúde, enquanto encontro de sujeitos..$^{10}$

Outro aspecto que aparece como indicador de vulnerabilidades socioeconômicas é a condição de moradia das famílias de crianças que se encontram hospitalizadas. Essa, muitas vezes, é precária ou, em alguns casos, ausente e, em muitos casos, somente é conhecida e detectada em profundidade, no momento da alta ou de uma visita domiciliar.

As condições deficitárias de moradia colocam os sujeitos em situação de pertença da exclusão social, a que muitas pessoas se encontram fadadas. A experiência de viverem tais processos de exclusão projeta-se nas representações dos sujeitos, de forma que as concepções de respeito e desrespeito são condicionadas pela maneira como elas os afetam. ${ }^{11}$

As falas a seguir nos mostram que o local onde as famílias e as crianças residem indicam situações de vulnerabilidades e podem se tornar determinantes para 0 sucesso de um tratamento, exigindo que a equipe lance seu olhar para outros aspectos, além do tratamento clínico, que poderiam passar despercebidos:

[...] Não ter uma casa adequada, úmida, fria [...] (E.8).

[...] o contexto onde ela vive, às vezes o porquê que esta criança está internando tanto. Claro que tem as implicações socioeconômicas... às vezes as condições de moradia são péssimas, com umidade, os familiares todos fumam dentro de casa, tem animais [....] (E.5).

[...] influencia desde aquele paciente que a gente sabe que mora ao lado do lixão e que vai ter que ter todo um apoio do serviço social, também do hospital, para que ele possa, depois de uma quimioterapia, neutropênico, voltar para casa. Eque casa é essa? Nós já tivemos pacientes que vão voltar para uma caixa de papelão [...] (E.1).

Se a qualidade da infância, por um lado, depende da família e suas relações, por outro, está profundamente marcada pelo lugar de moradia, pela vizinhança e pelos serviços disponíveis. A infância não remete apenas a um tempo na vida, mas também a um lugar. Lugar em que se residia, em que se situava a escola, a rua, a praça, uma vez que cada ambiente comporta formas de relacionamento e de comportamento que podem ser distintas. ${ }^{8}$

0 local de residência apresenta-se, para os enfermeiros, como um panorama das condições de enfrentamento e adequação das famílias em relação aos aspectos do tratamento; é o que nos ilustra a fala a seguir:

[...] temos que procurar conhecer de que forma eles vivem para poder adequar a informação, a orientação e tudo mais. No caso eu me lembrei de uma criança com diabete, que precisava de medicação e a geladeira mais próxima ficava na casa do vizinho e outro que precisava de oxigênio e não tinha rede elétrica [...]. Quer dizer, a criança conseguiu um aparelho que era super caro, mas não tinha luz para ligar, o que não adiantava em nada. Então isso faz com que a gente tenha que tomar outras atitudes [...] aí o cuidado de enfermagem acaba sendo maior [...] (E.9).

As condições de moradia transmitem aos profissionais de saúde a mensagem do tamanho do fardo que uma situação de doença pode representar. Quando se trata da doença de um filho, o fato de não possuir condições adequadas para the oferecer um suporte desencadeia uma cascata de sentimentos, como desesperança e desespero e agrava o quadro de miséria e desolação ao qual uma família está exposta. Como podemos observar a ação de "morar" possui várias significações, mas a principal se relaciona intimamente com a noção da dignidade humana.

Uma das provas mais evidentes da existência de uma família é o viver juntos sobre o mesmo teto. Isso significa que a noção de casa implica compartilhar um determinado modo de vida, o que pode ser denominado convivência familiar. ${ }^{12}$

Os componentes da vulnerabilidade individual que têm sido pontuados pelos estudiosos do tema são, fundamentalmente, de ordem cognitiva, representados pela quantidade e qualidade de informação que o indivíduo dispõe e a capacidade de elaborá-la, e de ordem comportamental, ou 
seja, pela capacidade e interesse para transformar atitudes em ações protegidas e protetoras. ${ }^{9}$

Portanto, no plano individual, considera-se que a vulnerabilidade a algum agravo está relacionada, basicamente, aos comportamentos que criam oportunidades para que as pessoas contraiam doenças. Esses comportamentos, associados à maior vulnerabilidade, não são entendidos e abordados neste quadro conceitual como uma decorrência imediata da ação voluntária das pessoas, mas estão relacionados tanto com condições culturais e sociais em que os comportamentos ocorrem como com o grau de consciência que as pessoas têm sobre tais comportamentos e o efetivo poder que podem exercer para transformá-los. ${ }^{10}$

Ao profissional enfermeiro, entre outras atribuições, compete educar para a promoção da saúde e a prevenção de agravos a ela. Na descoberta de um diagnóstico, no momento da alta hospitalar, ou mesmo em consultas rotineiras de puericultura, é preciso conhecer qual o grau de entendimento da familia sobre o que está se buscando aplicar naquele momento. Sentimentos como vergonha, frustração, medo, impotência, podem impedir os pais de revelarem o cenário real em que irá se desenvolver o cuidado à criança. Neste momento, estabelecer um vínculo de confiança é imprescindível, pois a compreensão inadequada de orientações pode significar grandes danos ao paciente.

Abaixo nos é apresentada, na expressão de uma das entrevistadas, a importância da manutenção de uma consonância entre as orientações dos profissionais e a assimilação destas pelos pais ou responsáveis na organização do cuidado à criança em situação de doença:

[...] E muitas vezes as dificuldades que eles encontram lá fora acabam atrapalhando. Sai com uma receita, por exemplo, de uma medicação. Aí, chega à farmácia e não tem aquela, tem outra e ele não sabe que aquele nome daquele remédio é 0 mesmo, tem o mesmo componente... Eles ficam com medo de comprar aquele ali, por não saber se vai fazer o mesmo efeito, ficam com vergonha de voltar com a receita e dizer que não conseguiram, em algumas situações acabam não continuando o tratamento em casa, por esse desconhecimento, que se parar a medicação vai interferir ou vai piorar. Muitas vezes, eles não fazem esta associação [...]. Ou muitas vezes a informação que a gente dá, na nossa linguagem, também é meio complicada, parece que eles entenderam, mas no segundo momento quando voltam no ambulatório, se vê que não tiveram aquele entendimento que tu achou que eles tinham, e aímuita coisa se perdeu no meio do caminho [...] (E.7).

0 cuidado à saúde faz parte da existência do ser humano, que, para crescer e se desenvolver de forma saudável, necessita ser cuidado ao longo de todas as etapas do seu ciclo vital. Porém a forma de cuidar é afetada pelos ambientes físicos, emocionais e culturais e pela estrutura social à qual o indivíduo pertence, uma vez que cada cultura possui suas próprias concepções de saúde, doença e cuidado. ${ }^{13}$

Educar para a saúde significa ser gerador de autoconfiança e autonomia nos sujeitos. É um ato perpassado por questões de sensibilidades, que preencha as lacunas de conhecimentos trazidas pelos indivíduos, favorecendo que descubram o que é preciso ser feito em relação ao tratamento de uma criança, como deve ser feito e quando irá ser feito. Conhecer a dinâmica do hospital e a organização dos cuidados que irão ser prestados possibilitam à família a redução do seu grau de ansiedade e a motivação para o desenvolvimento de uma atitude de colaboração. 0 trecho da fala a seguir nos exemplifica este cenário:

[...] 0 enfermeiro deve estar atento às necessidades individuais da criança, e da família como um todo. Então, neste sentido, exige um cuidado específico para cada situação. Devemos auxiliar a família no processo, para que ela mantenha um pouco de autonomia, já que a autonomia total é difíiil, pois ela está em situação vulnerável, em relação à doença, em relação a uma situação que ela não sabe como vai se desenrolar, como vai ser, se esta criança irá para casa, quais são as intercorrências [...] (E.6).

Entendendo-se que as questões de saúde/doença possuem componentes amplos e inter-relacionados, que não podem ser avaliados e tratados de forma isolada, sob pena de não se produzirem, de fato, recursos para a sua proteção, torna-se importante investir em outras formas de se pensar as intervenções em saúde. É por isso que estas vão encontrar eco, no que vem sido produzido e construído nos marcos do

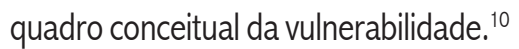

\section{Propostas e alternativas para o manejo das situações de vulnerabilidades socioeconômicas}

Realizar ações que mobilizem atores sociais na conquista de melhorias de situações de vulnerabilidades socioeconômicas é um desejo dos profissionais de enfermagem, que se exemplifica na sua luta diária para minimizar os efeitos da exclusão e da desigualdade que vitimizam nossa sociedade.

A situação socioeconômica é o fator que mais tem contribuído para a desestruturação da família, repercutindo 
diretamente e, de forma vil, nos mais vulneráveis deste grupo: às vítimas da injustiça social, da pobreza, da miséria, da falta de perspectiva e de um projeto existencial, que impõem a toda a família uma luta desigual e desumana pela sobrevivência. ${ }^{12}$

Este é o cenário em que as atitudes de transformação dos condicionantes de vulnerabilidades estão alocadas. Um quadro de miserabilidade, repleto de conflitos e dilemas, agravados, muitas vezes, pelo fato de se ter um filho hospitalizado, principalmente quando esta hospitalização acontece em virtude de uma patologia de prognóstico reservado.

0 desenvolvimento humano consiste em uma mudança duradoura na maneira pela qual uma pessoa percebe e lida com o seu ambiente. É o processo através do qual a pessoa adquire e desenvolve uma concepção mais ampliada do meio ambiente ecológico e se torna mais motivada e capaz de se envolver em atividades que revelem suas propriedades. ${ }^{2}$ Esta temática propicia o estabelecimento da relação entre desenvolvimento e ambiente; logo, para que a criança se desenvolva de forma saudável, é necessário conhecer onde e como ela vive.

Nesta perspectiva encontram-se as visitas domiciliares, vistas pelos entrevistados como marco inicial para o diagnóstico da situação de enfrentamento das vulnerabilidades das famílias, como colocado na seguinte fala:

\section{[...] Outras estratégias são as visitas domiciliares, eu acho que tu conhecendo bem a família e todo o contexto social dela, tu consegue ajudar, tu consegue melhorar este atendimento porque se trabalha direto com as famílias e as situações de vulnerabilidade. Eu trabalho há muito tempo neste hospital, e aqui a gente sempre apreendeu a trabalhar bem nas vulnerabilidades, bem nos problemas da família. Eu acredito que a gente deva estimular mais a visita domiciliar. Eu acho isso bem importante [...] a gente já a realiza de alguma forma $[\ldots](E .4)$.}

A visita domiciliar se constitui em alternativa de cuidado que interliga saberes, propicia a escuta e a valorização do outro, não se constituindo em momentos de transmissão de quem sabe para quem aprende no estabelecimento de relações de poder. Apesar de a ação educativa na visita domiciliar ser referida como positiva, em alguns momentos, também se evidencia a sua realização através de uma prática profissional impositiva, que desvaloriza conhecimentos e decisões em torno do cuidado à saúde. ${ }^{14}$
0 desafio para a visita é concretizar, a partir da permissão de processos de diálogo e de respeito aos conhecimentos, os valores e práticas das famílias. A prática de visita domiciliar se constitui em uma tecnologia de cuidado, e desta forma necessita de aperfeiçoamento constante, e da utilização de sensibilidade, aliada à competência técnica.

Diversos são os sentimentos que mobilizam pessoas a ajudar o outro, entre eles destaca-se a compaixão. A equipe de enfermagem não fica alheia a esses sentimentos. 0 fato de uma criança, que não tenha meios adequados de subsistência, adentrar a unidade de internação provoca uma mobilização em torno daquela família, no sentido de auxiliá-la e transmitirIhe conforto e compreensão. É o que nos é salientado no discurso abaixo:

[...] o paciente SUS aqui é muito bem atendido e, muitas vezes, quanto mais carente ele é, mais acaba sendo, parece, acolhido aqui. Na nossa Unidade aqui da pediatria, parece que quanto mais a criança tem todo esse contexto social, mais ele é abraçado por toda equipe, é uma coisa incrivel [...] os pacientes chegam aqui e daqui a pouco estão cheios dos presentes, se não tem roupa, sem falar, já está com o guarda-roupa cheio. Sai com um monte de presentes, montes de brinquedos, suprimentos, assim, que são típicos do humanismo de cada pessoa, individual. É uma coisa que acaba espontaneamente acontecendo $[\ldots](E .9)$.

Outras proposições de promoção à saúde, que foram destacadas por alguns profissionais como importantes para manutenção do crescimento e desenvolvimento da criança e para resolução de dificuldades específicas dos pais, são 0 atendimento ambulatorial e a consulta de enfermagem. Essas aparecem como momento de destaque, pois possibilitam fornecimento de atenção adequada principalmente na pósalta dos pacientes, como identificamos nas falas a seguir:

[...] Uma coisa que está sendo um sonho e está se tornando realidade e vai auxiliar a diminuir uma dificuldade que os pacientes têm encontrado, é criar um ambulatório que atenda [...] os pacientes que tem alta, dependendo de algum tipo de tecnologia, que fazem uso de alguma sonda vesical contínua, de usar um cateter que requer cuidados permanentes como PIC, que necessita curativo e revisão semanal. Hoje isso é uma dificuldade muito 
grande para os pacientes e quanto mais carente pior. Eu acho que se a gente formar este ambulatório a gente vai estar suprindo mais esta lacuna, vamos estar facilitando um tratamento que já é longo e doloroso destes pacientes crônicos [...] (E.1).

\section{[...] Eu, por exemplo, procuro sempre encaminhar} as crianças com asma brônquica lá para o grupo do ambulatório que fazem a orientação. [...] A família é receptiva à marcação de consultas para aprender sobre a asma brônquica, como ela funciona, porque acontece, o que a gente pode fazer para prevenir. $E$ as pessoas de um modo geral são bem receptivas nesta parte [...] (E.2).

0 momento da consulta de enfermagem propicia um adequado acompanhamento de diversas questões da criança e sua família, em especial as relativas ao enfrentamento das situações de doenças. A atenção ao desenvolvimento da criança enfatiza a dedicação e a competência, sendo realizada com profissionalismo e humanidade. A atuação da enfermeira se encontra entre 0 desenvolvimento das potencialidades da criança e as limitações que cada família, ao adentrar o consultório mensalmente, traz para serem trabalhadas. Nesse caso, a enfermagem convive com diferentes necessidades. Por isso, o planejamento da atenção à saúde da criança envolve aspectos biológicos, econômicos, sociais e psicológicos junto às famílias que desenvolvem os cuidados, visando melhorar a qualidade de vida de seus pequenos pacientes. ${ }^{15}$

Ao realizar 0 atendimento de uma necessidade específica de uma criança e sua família, como a realização do acompanhamento de uma patologia crônica, o profissional enfermeiro mobiliza conhecimentos, que traduzem sua competência técnico-científica no estabelecimento de um plano de cuidados, que, por sua vez, somente é efetivo no momento que alia às suas atividades o caráter humanístico, necessário às condutas pertinentes ao trabalho com seres humanos.

A devida atenção voltada aos familiares se torna necessária no sentido de minimizar angústias vivenciadas em situações de estresse. Algumas condições são mais dificilmente observadas e exigem sensibilidade e afinidade da equipe de enfermagem para detectá-las, pois podem ser expressas por sentimentos muitas vezes não verbalizados. Portanto, estes sentimentos precisam ser percebidos pelos profissionais, pois irão facilitar a prestação de um cuidado integral. ${ }^{16}$

Nesse contexto éque a consulta de enfermagem, enquanto ato privativo do enfermeiro, adquire fundamental importância. A enfermeira, ao se aproximar da realidade de cada família, identifica necessidades e dificuldades, estabelecendo uma relação de confiança, proporcionando ajuda efetiva. Esta situação é corroborada pelo fato de a familia e suas necessidades serem alicerces do cuidado. Todos os integrantes são convidados pela enfermeira a participarem dos cuidados com a criança, possibilitando um ambiente acolhedor efavorável ao crescimento e desenvolvimento desta.

0 ambiente criado pela enfermeira, no momento do atendimento, expressa a preocupação com o bem-estar da criança, transmitindo interesse, aceitação e disponibilidade. A enfermeira destaca o outro como pessoa única e torna o encontro pessoal ao se apresentar à criança e seus familiares, ao cumprimentá-los e, sempre a cada encontro, identificar mudanças percebidas neles. ${ }^{17}$

\section{CONCLUSÕES}

Ao concluirmos esta pesquisa, observamos muitos aspectos que permeiam o cenário das vulnerabilidades socioeconômicas no cotidiano de cuidado das crianças hospitalizadas e suas famílias. As enfermeiras trazem exemplos práticos de vulnerabilidades socioeconômicas com as quais convivem durante a prestação da assistência de enfermagem direta à criança e sua família e como estes estão postos na vida das mesmas, sendo reconhecidos, por exemplo, pela capacidade cognitiva das pessoas, a forma de acesso aos serviços de saúde, entre outros.

Contemplando o objetivo desta pesquisa, ocorreu uma aproximação das questões conceituais das vulnerabilidades e do meio ambiente ecológico com a prática assistencial cotidiana em pediatria. As manifestações das vulnerabilidades socioeconômicas e as alternativas para minimizar os seus efeitos possibilitaram conhecer o entendimento que as enfermeiras possuem sobre as influências destas na vida de crianças e de suas famílias.

A compreensão da importância do trabalho de enfermagem se fez presente ao longo da análise dos resultados. É de fundamental importância que o profissional enfermeiro situe sua atuação no cenário das vulnerabilidades socioeconômicas que acometem as famílias de crianças em situações de enfermidade. Cada olhar, cada gesto ou palavra, que emanam da díade criança-família, são repletos de significados que indicam o caminho a ser seguido, conhecido por muitas das enfermeiras entrevistadas.

Para o cenário de atuação de enfermagem pediátrica, muitas foram as colaborações oriundas das falas das entrevistadas. Pode-se verificar que, por meio de atividades desenvolvidas e em projeto de desenvolvimento, o profissional enfermeiro possui condições de estabelecer ações efetivas que 
favoreçam o crescimento e desenvolvimento saudáveis da criança enferma e sua família, em situações de vulnerabilidades socioeconômicas. Tais atitudes estão representadas nas falas das participantes pela verificação da história familiar, visita domiciliar, consulta de enfermagem, utilização de recursos da comunidade, entre outros, e, que quando implementadas, valorizam e engrandecem o trabalho de toda a equipe de enfermagem.

A demonstração de preocupação com o contexto em que cada família vive e desenvolve cuidados à saúde é valorizada pelos enfermeiros, que, com seus relatos, possibilitaram que esta pesquisa se transformasse em momento de aquisição de conhecimentos e reflexão acerca da temática. Espera-se que este estudo seja base para próximos e contribua com todos os profissionais para a melhoria da prática assistencial de enfermagem pediátrica.

\section{REFERÊNCIAS}

1. Ayres JRCM. Vulnerabilidade e avaliação de ações preventivas: HIV/ AIDS e abuso de drogas entre adolescentes. São Paulo (SP): Faculdade de Medicina da USP; 1996.

2. Bronfenbrenner U. A ecologia do desenvolvimento humano: experimentos naturais e planejados. Porto Alegre (RS): Artes Médicas; 1996.

3. Ciampone MH, Tonete VLP, Pettengill MAM, Chubaci RYS. Representações sociais da equipe de enfermagem sobre a criança desnutrida e sua família. Rev Latino-am Enfermagem 1999 jul; 7(3): 99-106.

4. Pedroso MLR, Motta MGC. Influências das vulnerabilidades sócioeconômicas no cuidado às crianças em unidades de internação pediátricas: a visão do enfermeiro. [monografia de conclusão de curso de especialização]. Porto Alegre (RS): Escola de Enfermagem/ UFRGS; 2008.

5. Polit DF, Beck CT, Hungler BP. Fundamentos de pesquisa em enfermagem: métodos, avaliação e utilização. $5^{\mathrm{a}}$ ed. Porto Alegre (RS): Artmed; 2004.

6. Minayo MCS, organizadora. Pesquisa social: teoria, método e criatividade. $26^{\text {a }}$ ed. Rio de Janeiro (RJ): Vozes; 2007.

7. Muñoz Sánchez Al, Bertolozzi MR. Pode o conceito de vulnerabilidade apoiar a construção do conhecimento em saúde coletiva? Cienc Saude Colet 2007 abr; 12(2): 319-24.

8. Sierra VM, Mesquita WA. Vulnerabilidades e fatores de risco na vida de crianças e adolescentes. São Paulo Perspec 2006 jan/mar; 20(1): 148-55.
9. Ayres JRCM, França Júnior I, Calazans GJ, Saletti Filho HC. 0 conceito de vulnerabilidade e as práticas de saúde: novas perspectivas e desafios. In: Czeresnia D, Freitas CM, organizadores. Promoção da saúde: conceitos, reflexões, tendências. Rio de Janeiro (RJ): FIOCRUZ; 2003. p. 117-39.

10. Meyer DE, Mello DF, Valadão MM, Ayres JRCM. Você apreende, a gente ensina? Interrogando relações entre educação e saúde desde a perspectiva da vulnerabilidade. Cad Saude Publica. 2006 jan/jun; 22(6): 1335-342.

11. Lopes JR. Processos sociais de exclusão e políticas públicas de enfrentamento da pobreza. Cad CRH. 2008 maio/ago; 21 (53): 34963.

12. Gomes MA, Pereira MLD. Família em situação de vulnerabilidade social: uma questão de políticas públicas. Cienc Saude Colet 2005 abr/jun; 10(2): 357-63.

13. Zanatta EA, Motta MGC. Saberes e práticas de mães no cuidado à criança de zero a seis meses. Rev Gaucha Enferm 2007 dez; 28(4): 556-63.

14. Mandú ENT, Gaíva MAM, Silva MA, Silva AMN. Visita domiciliária sob o olhar de usuários do Programa de Saúde da Família. Texto\&Contexto Enferm 2008 jan/mar; 17(1): 131-40.

15.Pedroso MLR. Programa Prá-Nenê: percepções acerca da educação em saúde realizada pela enfermeira em uma unidade básica de saúde [trabalho de conclusão de curso]. Porto Alegre (RS): Escola de Enfermagem/UFRGS; 2006.

16. Monteiro MAA, Pinheiro AKB, Souza AMA. Vivências de Puérperas com Filhos Recém-Nascidos Hospitalizados. Esc Anna Nery Rev Enferm 2007 jun; 11 (2): 276-82.

17. Sigaud CHS, Verissimo MLOR. Enfermagem pediátrica: o cuidado de enfermagem à criança e ao adolescente. São Paulo (SP): EPU; 1996.

Data de recebimento: 25/03/09 Data de reapresentação 20/10/2009 Data de aprovação 04/02/2010 